\title{
In Situ Forming Injectable Hydrogel For Encapsulation Of Nanoiguratimod And Sustained Release Of Therapeutics
}

This article was published in the following Dove Press journal: International Journal of Nanomedicine

\author{
Zhenzhen $M a^{1, *}$ \\ Cheng $\mathrm{Tao}^{2, *}$ \\ Lin Sun' \\ Shengbei $\mathrm{Qi}^{2}$ \\ Yuan Le $\mathrm{e}^{2,3}$ \\ Jiexin Wang ${ }^{2-4}$ \\ Changhong $\mathrm{Li}^{1}$ \\ Xiangyuan Liu' \\ Jianjun Zhang ${ }^{2}$ \\ Jinxia Zhao'
}

'Department of Rheumatology and Immunology, Peking University Third Hospital, Beijing 10019I, People's Republic of China; ${ }^{2}$ College of Chemical Engineering, Beijing University of Chemical Technology, Beijing 100029,

People's Republic of China; ${ }^{3}$ Research Center of the Ministry of Education for High Gravity Engineering and Technology, Beijing University of Chemical

Technology, Beijing 100029, People's Republic of China; ${ }^{4}$ Beijing Advanced Innovation Center for Soft Matter Science and Engineering, Beijing University of Chemical Technology, Beijing 100029, People's Republic of China

*These authors contributed equally to this work
Correspondence: Jianjun Zhang; Jinxia Zhao

Email zhangij@mail.buct.edu.cn; zhao-jinxia@I63.com
Background: Iguratimod (IGUR) is a novel disease-modifying antirheumatic drug used for treating rheumatoid arthritis (RA). To improve its bioavailability and to alleviate gastrointestinal side effects, we changed the formulation into nanoiguratimod-loaded hydrogel (NanoIGUR-loaded hydrogel) composites for sustained release of therapeutics.

Methods: IGUR was first encapsulated in biodegradable polyvinyl alcohol micelle by liquid antisolvent precipitation (LAP) technology, and then loaded into an in situ injectable hyaluronic acid hydrogel, which was cross-linked by PEG (Thiol) ${ }_{2}$ (HS-PEG-SH) through Michael addition reaction. In vitro, the biological effects (proliferation, migration, and invasion) of NanoIGURloaded hydrogel on fibroblast-like synoviocytes (RA-FLS) from RA patients were evaluated. In vivo, the pharmacokinetics of NanoIGUR-loaded hydrogel was assessed and the efficacy of NanoIGUR-loaded hydrogel in treating collagen-induced arthritis (CIA) rats was evaluated.

Results: By the LAP technique, we acquired the amorphous form nanoiguratimod, with an average size of $458 \mathrm{~nm}$, which had higher dissolution rates and higher stability. The release of IGUR from hydrogel composite in PBS was gradual and sustained for up to $72 \mathrm{hrs}$ compared with nanoiguratimod. Different concentrations of NanoIGUR-loaded hydrogel inhibited the proliferation, migration, and invasion of RA-FLS. The pharmacokinetic parameters showed better bioavailability and longer half-life time with NanoIGUR-loaded hydrogel by subcutaneous administration than oral raw iguratimod. Animal experiments confirmed that subcutaneous injection of NanoIGUR-loaded hydrogel $(10 \mathrm{mg} / \mathrm{kg}$ every 3 days) and oral raw iguratimod $(10 \mathrm{mg} / \mathrm{kg}$ daily) showed similar efficacy in decreasing arthritis index score, pathological score, and expression of inflammatory cytokines.

Conclusion: Overall, we demonstrate that NanoIGUR-loaded hydrogel provides a new route of administration and extends the administration interval. It could be a promising drug-delivery approach in the management of RA.

Keywords: iguratimod, nanoparticles, injectable hydrogel, rheumatoid arthritis, drug sustained-release system

\section{Introduction}

Rheumatoid arthritis (RA) is a chronic inflammatory and autoimmune disease characterized by symmetrical, progressive, and aggressive polyarthritis. ${ }^{1}$ The disease invades articular cartilage and surrounding soft tissues, resulting in the destruction of articular cartilage, bone, and joint capsule, eventually leading to joint deformity, physical disability, and loss of function, which significantly reduces the quality of life of patients. . $^{2,3}$ 
Early treatment is especially important for the prognosis of RA. ${ }^{4}$ Drugs for treating RA mainly include nonsteroidal anti-inflammatory drugs (NSAIDs), disease-modifying antirheumatic drugs (DMARDs), glucocorticoids, and biological agents. Traditional DMARDs, including leflunomide, methotrexate (MTX), and sulfasalazine, have been widely used in clinical practice and show a certain degree of therapeutic efficacy. However, there is still a significant proportion of patients with RA who do not achieve clinical remission with these traditional DMARDs. ${ }^{5}$ Among biologics commonly used in RA, tumor necrosis factor alpha (TNF- $\alpha$ ) inhibitors were the most frequently used; however, about $20 \%$ to $40 \%$ of RA patients treated with a TNF- $\alpha$ inhibitor fail to achieve a 20\% improvement in American College of Rheumatology (ACR20) criteria, and moreover lose response over time, due to secondary failure or acquired therapeutic resistance, ${ }^{6}$ and some may experience adverse events such as infections. ${ }^{7}$ Moreover, high cost also makes it difficult to achieve wide applications in the clinic. ${ }^{8}$ Although the efficacy of new dosage forms of traditional DMARDs, like MTX, has been investigated in a few studies, the results have not been translated into clinical practice. ${ }^{9,10}$

Iguratimod (IGUR), a methanesulfonanilide, also known as T-614 [N-(3-formylamino-4-oxo-6-phenoxy-4H-1-benzopyran-7-yl)], was exclusively developed in countries of the Asia-Pacific region and has been shown to possess antiinflammatory properties. ${ }^{11}$ As a novel DMARD, IGUR is effective in treating RA; however, side effects, such as elevated liver enzymes and gastrointestinal discomforts, are the main concerns in its clinical applications. ${ }^{12}$ IGUR is practically insoluble in aqueous solvents and is only available in oral dosage forms. ${ }^{11}$ A major problem with oral administration is that the speed and extent of drug absorption are affected by multiple factors, owing to the complex physiological state of the gastrointestinal tract. The low solubility of drugs is responsible for incomplete absorption and low bioavailability, which affects their clinical efficacy and increases its gastrointestinal side effects. ${ }^{13}$ To alleviate the side effects of IGUR, and to improve its bioavailability and promote its application in the treatment of RA, it is necessary to develop a convenient and effective drug-delivery system for this drug.

In this study, we developed an in situ forming injectable hydrogel drug-delivery system, which was used as a depot of nanoiguratimod (NanoIGUR), for the treatment of RA. We evaluated the biological effects (proliferation, migration, and invasion) of the NanoIGUR-loaded hydrogel on fibroblastlike synoviocytes (RA-FLS). The pharmacokinetics of oral raw IGUR and subcutaneous injection of NanoIGUR-loaded hydrogel was assessed in healthy rats. Furthermore, the efficacy of NanoIGUR-loaded hydrogel in treating collageninduced arthritis (CIA) rat model was evaluated. Overall, we reported the successful preparation of a new sustainedrelease NanoIGUR-loaded hydrogel.

\section{Materials And Methods Materials}

The raw IGUR was provided by the Simcere Pharmaceutical Group (Jiangsu, China). Adipic acid dihydrazide (ADH, $>99.0 \%$ ), 1-ethyl-3-(3-dimethylaminopropyl) carbodiimide hydrochloride (EDC, 98\%), triethanolamine (TEA, 98\%), and polyvinyl alcohol (PVA; average $\mathrm{Mw} 31,000)$ were purchased from Shanghai Aladdin Bio-Chem Technology Co. Ltd. (Shanghai, China). Dimethyl sulfoxide (DMSO, AR grade), used as a solvent, was purchased from Beijing Chemical Works (Beijing, China). Sodium hyaluronan (HA; average Mw 50,000) was purchased from Bloomage Freda Biopharm Co. Ltd. (Jinan, China). N-Acryloxysuccinimide (NHS-Ac, 99\%) and 4-(2-hydroxyethyl) piperazine-1-ethanesulfonic acid (HEPES, 99\%) were purchased from J\&K Scientific Ltd. (Beijing, China). PEG (Thiol) $)_{2}$ (HS-PEG-SH), with an average $\mathrm{Mw}$ of 3500, was purchased from JenKem Technology Co. Ltd. (Beijing, China). All the compounds were used as received.

\section{Hyaluronic Acid-Acrylate Modification}

Sodium hyaluronan was modified to contain acrylate functionalities as previously described. ${ }^{14}$ Briefly, as shown in Figure 1A, HA $(2.00 \mathrm{~g}, 50 \mathrm{kDa}, 5.28 \mathrm{mmol}$ carboxylic acid) was reacted with $25.0 \mathrm{~g}$ (143.5 mmol) of ADH at $\mathrm{pH}$ 4.75 in the presence of $4.0 \mathrm{~g}(20 \mathrm{mmol})$ of EDC overnight and purified by dialysis [8000 molecular weight cutoff (MWCO)] in deionized (DI) water for 5 days. The purified intermediate (HA-ADH) was lyophilized and stored at $-20^{\circ} \mathrm{C}$ until used. Approximately $45 \%$ of the carboxyl groups were modified with $\mathrm{ADH}$; the modification was characterized using $1 \mathrm{H}$ NMR $\left(\mathrm{D}_{2} \mathrm{O}\right)$ by taking the ratio of peaks at $\delta=1.54$ and 2.26, corresponding to the eight hydrogens of the methylene groups on the $\mathrm{ADH}$, to the singlet peak of the acetyl methyl protons in HA $(\delta=1.88)$ (Figure 1B). All the modified HA-ADH was reacted with NHS-Ac (4.46 g, $26.38 \mathrm{mmol})$ in HEPES buffer $(10 \mathrm{mM}$ HEPES, $150 \mathrm{mM} \mathrm{NaCl}, 10 \mathrm{mM}$ EDTA, pH 7.2) overnight and purified by dialysis in a $100-0 \mathrm{mM}$ salt gradient for 1 day, followed by dialysis in DI water for 3-4 days before 
A

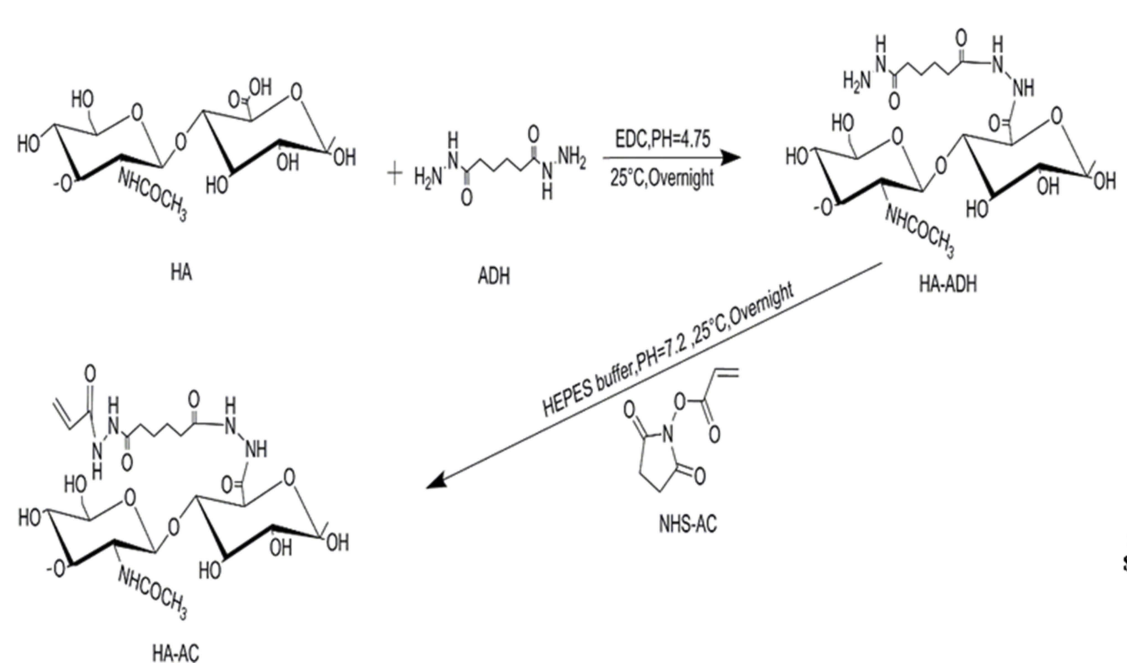

B

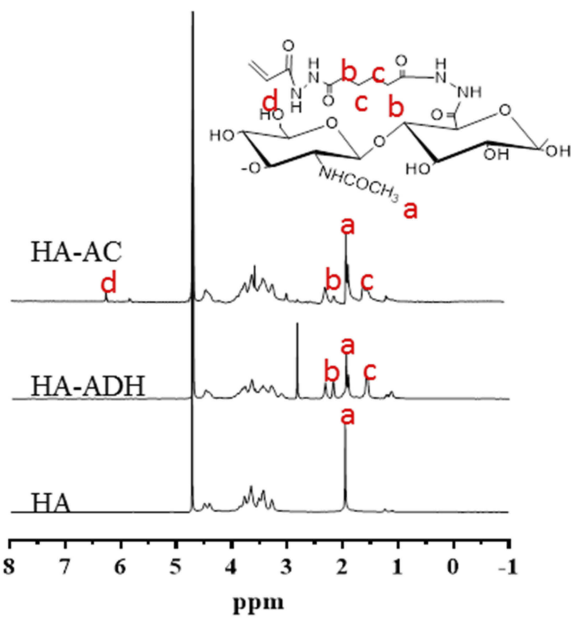

Figure I Synthesis scheme for hyaluronic acid-acrylate (HA-AC) (A) and the results of NMR (B). a:- $\mathrm{CH}_{3}$, b:- $-\mathrm{CH}_{2}-\mathrm{CO}-, \mathrm{c}:-\mathrm{CH}_{2}$, and d: $-\mathrm{CH}$.

lyophilization. The degree of acrylation was determined to be $\sim 11 \%$ using $1 \mathrm{H}$ NMR $\left(\mathrm{D}_{2} \mathrm{O}\right)$ by taking the ratio of the multiplet peak at $\delta=6.2$, corresponding to the cis and trans acrylate hydrogens to the singlet peak of the acetyl methyl protons in HA $(\delta=1.88)$ (Figure 1B). Dynamic mechanical thermal analysis (DMTA-V, Rheometric Scientific, USA) was used to analyze the loss modulus (E') and storage modulus (E') of NDHM, and the tests were run from $0.01 \mathrm{~Hz}$ to $10 \mathrm{~Hz}$ at $37{ }^{\circ} \mathrm{C}$.

\section{Preparation Of NanolGUR By Liquid Antisolvent Precipitation}

NanoIGUR, with high aqueous phase dispersibility, was synthesized using the liquid antisolvent precipitation (LAP) method. Briefly, $20 \mathrm{mg}$ of the raw IGUR and $20 \mathrm{mg}$ of PVA were dissolved in $1 \mathrm{~mL}$ of DMSO as the drug solution. One milliliter of the drug solution was quickly added to $20 \mathrm{~mL}$ of ultrapure water (antisolvent), and then the mixture was stirred for 30 mins. After dialysis and lyophilization, the powder was stored at $4^{\circ} \mathrm{C}$ for further use.

\section{Characterizations Of NanolGUR}

The particle size of NanoIGUR was determined by dynamic light scattering (Nano ZS, Malvern Instruments, $\mathrm{UK}$ ) and reported as the Z-average (intensity-based mean particle diameter). The measurements were repeated three times. The morphology of the samples was examined using a transmission electron microscope (TEM, Hitachi HT7700, Japan). The drug-loading capacity (DLC) and dissolution rate of the sample were determined using a
UV/visible spectrophotometer (Varian Cary 50, USA) at a wavelength of $309 \mathrm{~nm}$, according to the formula:

DLC (wt. \%) = (weight of the drug/weight of drugpolymer nanoparticles $) \times 100$

\section{Evaluation Of In Vitro Drug Release}

The dissolution rate of raw IGUR and NanoIGUR in PBS buffer $(150 \mathrm{mM}, \mathrm{pH} 7.4)$ at $37^{\circ} \mathrm{C}$ was determined. In each experiment, $1.0 \mathrm{mg}$ of the sample was mixed with $5 \mathrm{~mL}$ of PBS buffer and was fully dispersed. The mixture was then transferred into a dialysis bag (3500 MWCO), and the bag was put into a tube containing $20 \mathrm{~mL}$ of PBS buffer. Further, $3 \mathrm{~mL}$ of PBS buffer was withdrawn from the tube at predetermined periods of time, and $3 \mathrm{~mL}$ of fresh buffer was added into the tube. The dissolution rate of raw IGUR and NanoIGUR was calculated by determining the UV absorption of IGUR at $309 \mathrm{~nm}$. The NanoIGURloaded hydrogel was prepared by first dissolving the polymers, HA-AC and HS-PEG-SH, using the NanoIGUR solution. Subsequently, equivalent volumes and concentrations of the two solutions were mixed for allowing the gelation. The process for the solidification of NanoIGURloaded hydrogel is shown in Figure $2 \mathrm{C}$. The resulting hydrogels $(250 \mu \mathrm{L})$ were loaded into a dialysis bag (3500 MWCO), and the dialysis bag was put into a tube containing $20 \mathrm{~mL}$ of PBS buffer. Further, $3 \mathrm{~mL}$ of PBS buffer was withdrawn from the tube at predetermined periods of time, and $3 \mathrm{~mL}$ of fresh buffer was added into the tube. The dissolution rate of IGUR from the hydrogel was calculated by determining the UV absorption of IGUR at $309 \mathrm{~nm}$. 
A<smiles>C#CS(=O)(=O)Nc1cc2occ(NC=O)c(=O)c2cc1Oc1ccccc1</smiles>
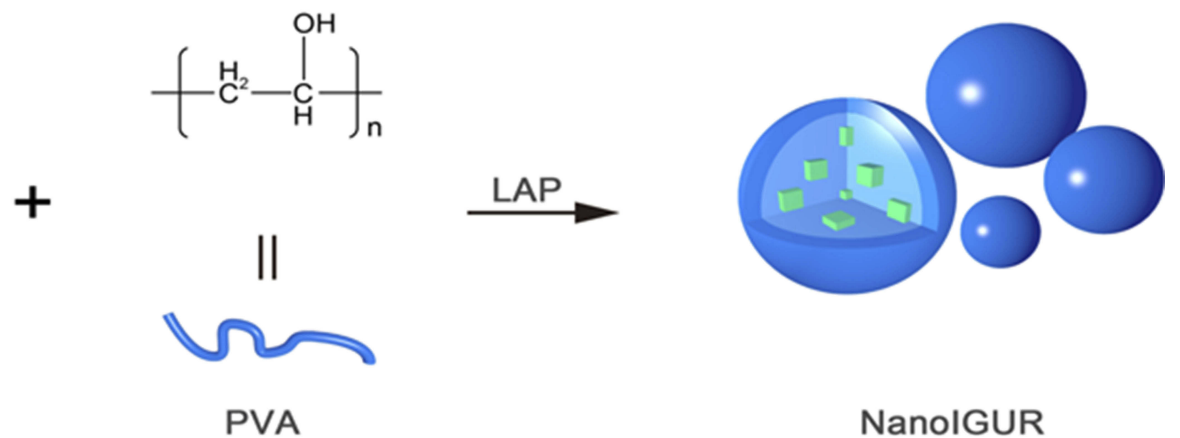

Iguratimod(IGUR)

B
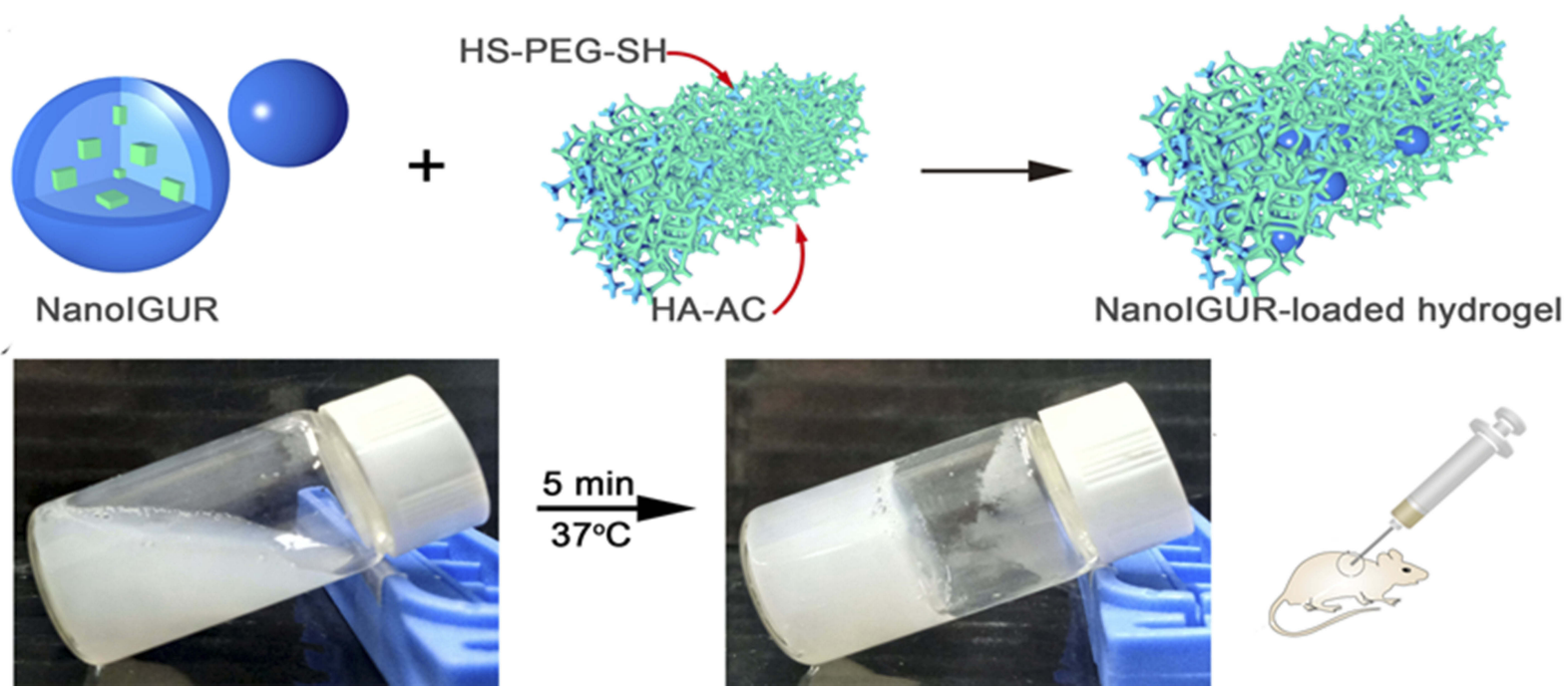

Figure 2 Schematic illustration showing the process for preparation of NanolGUR (A); the process for preparation of NanolGUR-loaded hydrogel (B); and the solidification process of NanolGUR-loaded hydrogel and the administration of subcutaneous injection of NanolGUR-loaded hydrogel to the collagen-induced arthritis (CIA) rat (C).

\section{Cell Studies}

\section{Cell Culture}

Synovial specimens were taken from five patients with RA who underwent synovectomy or joint replacement at Peking University Third Hospital. All the patients met the 2010 ACR/ EULAR classification criteria for RA. ${ }^{15}$ RA-FLS were isolated from the fresh synovial tissues as previously described. ${ }^{16}$ After the synovial tissue was obtained, the excess adipose tissue was removed immediately, and the synovial tissue was infiltrated into high glucose DMEM (GIBCO-BRL) with 15\% FBS (GIBCO-BRL), $100 \mathrm{U} / \mathrm{mL}$ penicillin (GIBCO), and 100 $\mu \mathrm{g} / \mathrm{mL}$ streptomycin (GIBCO) after three times of irrigation with PBS. The synovial tissue was cut into tissue fragments about $2 \mathrm{~mm} \times 2 \mathrm{~mm}$ in size and then inoculated in the flask and added $2 \mathrm{~mL}$ DMEM containing 20\% FBS. The flask was reversed after incubation at $37^{\circ} \mathrm{C}$ and $5 \% \mathrm{CO}_{2}$ incubator for 6-10 hrs. Primary cell growth could be seen after 5-7 days and RA-FLS from passages three to six was used in this study. This study was conducted in accordance with the ethical principles originating in the Declaration of Helsinki (2004 revision) and was approved by the Ethics Committee of Peking University Third Hospital (No. LA2015044-02), and all the patients provided signed informed consent.

\section{Vitro MTS Assay}

In addition to the blank controls (10\% FBS high glucose DMEM complete medium) and the sham controls (complete medium containing PBS for the raw IGUR or blank gel for the NanoIGUR-loaded hydrogel), different concentrations $(40,80,160$, and $320 \mu \mathrm{g} / \mathrm{mL})$ of raw IGUR or NanoIGURloaded hydrogel were used for cell proliferation experiments. Cells were seeded $\left(3 \times 10^{3}\right.$ cells $\left./ 100 \mu \mathrm{L}\right)$ and incubated overnight to allow cell adherence. Six duplicate wells were assigned for each group. After $48 \mathrm{hr}$ incubation with different concentrations of raw IGUR or NanoIGUR-loaded hydrogel, $20 \mu \mathrm{L}$ MTS was added to each well. Subsequently, after further incubation for $2 \mathrm{hrs}$, the absorbance (OD) was measured at $490 \mathrm{~nm}$ and analyzed statistically. 


\section{Vitro Migration Assay}

The transwell plate was pre-balanced before the migration experiment and the drug concentration gradient was set as in the MTS assay. RA-FLS in serum-free medium $\left(3 \times 10^{3}\right.$ cells $/ 100 \mu \mathrm{L}$ ) was added to the upper chamber of $8-\mu \mathrm{m}$ pore size transwell chambers (Corning). The bottom chambers contained $10 \%$ FBS as a chemoattractant, and different concentrations of raw IGUR or NanoIGUR-loaded hydrogel were separately added into the upper chambers. Cells were allowed to migrate through the porous membrane for $48 \mathrm{hrs}$ at $37^{\circ} \mathrm{C}$. Nonmigrating cells that remained on the upper surface of the filter were removed with cotton swabs, and the remaining cells on the lower surface of the filter were fixed with $600 \mu \mathrm{L} 4 \%$ paraformaldehyde at room temperature for 30 mins, stained with $600 \mu \mathrm{L} 0.1 \%$ crystal violet and counted under a bright field microscope (Nikon E400). Five visual fields were randomly selected for counting, and each experiment was independently performed three times.

\section{Vitro Invasion Assay}

The matrigel matrix (Invitrogen) was converted into a liquid state before the invasion experiment. The matrix glue was diluted with precooled serum-free medium (medium: matrix glue $=5: 1)$ and $60 \mu \mathrm{L}$ of it was added to each compartment. Incubation was done overnight in an incubator. The remaining steps were the same as in the migration experiments. Five visual fields were randomly selected for counting and three independent replications of the experiment were conducted.

\section{Animal Studies}

\section{Animals}

The animal study was approved by the Peking University Animal Protection and Ethics Committee (No. LA201425) and strictly abided by the Laboratory Animal Protection and Use guidelines. Female Lewis rats $(150 \pm 10 \mathrm{~g}, 6$ weeks) were purchased from Vital River Laboratory Animal Technology Co. Ltd (Beijing, China). Prior to the experiments, all the animals were housed in specific pathogen-free animal rooms under controlled temperature (23 \pm $2^{\circ} \mathrm{C}$ ) and $12-\mathrm{hr}$ light/dark cycles for at least 7 days and had free access to diet and water.

\section{Vivo Pharmacokinetics}

Normal female Lewis rats were used for studying the pharmacokinetics of IGUR. The drug concentration in plasma was determined by high-performance liquid chromatography (HPLC) after administration of a single dose $(10 \mathrm{mg} / \mathrm{kg})$ of IGUR. The rats in the raw IGUR group were orally administered raw IGUR dissolved in $0.5 \%$ carboxymethyl-cellulose (CMC) sodium in distilled water. Serial blood samples were collected in heparinized tubes at 10 and 30 mins, and at 1, 2, $3,4,6,8,12,18$, and 24 hrs. The rats in the NanoIGURloaded hydrogel group were subcutaneously injected with NanoIGUR-loaded hydrogel and blood samples were collected at 10 and 30 mins, and at 1, 2, 3, 4, 6, 8, 12, 18, 24, 48, 72,96 , and $120 \mathrm{hrs}$ in heparinized tubes, as described by Xiaofeng Guo et al. ${ }^{17}$ The pharmacokinetic parameters were processed by DAS 2.0 software and calculated according to the characteristics of the pharmacokinetic-time curve.

\section{Collagen-Induced Arthritis (CIA) Model}

CIA is an ideal animal model for RA. ${ }^{18}$ To induce CIA, bovine type II collagen (Chondrex, WDC, USA, 20,022) was dissolved in an equal volume of Freund's complete adjuvant (Chondrex, WDC, USA, 7001), and a total volume containing $400 \mu \mathrm{g}$ collagen was subcutaneously injected to each rat. Seven days later, immunization was repeated with bovine type II collagen and incomplete Freund's adjuvant (Chondrex, WDC, USA, 7002). After 14 days, the incidence of arthritis was observed and recorded daily. The severity of CIA joint inflammation was evaluated as the arthritis index (AI) score. ${ }^{19}$ Briefly, the severity of arthritis in each paw was rated from 0 (normal) to 4 (severe). The AI score of the onset joint was added, with a maximum score of 16 . The scoring was performed independently by two researchers.

\section{Vivo Therapeutic Efficacy}

The CIA rats were randomly allocated to three groups. No intervention was done in one group (CIA model, $\mathrm{n}=7$ ), whereas rats in the other two groups received the drugs as follows: raw IGUR $10 \mathrm{mg} / \mathrm{kg}$ intragastrically, daily (raw IGUR, $\mathrm{n}=7$ ) and the NanoIGUR-loaded hydrogel $10 \mathrm{mg} / \mathrm{kg}$ by subcutaneous injection, every 3 days (NanoIGUR-loaded hydrogel, $n=7$ ). The healthy normal rats were used as healthy controls (healthy control, $\mathrm{n}=7$ ). In this study, $0.5 \% \mathrm{CMC}$ was used as a suspension agent for raw IGUR to increase the distribution area of IGUR in the gastrointestinal tract after oral administration, and made it absorb thoroughly. Experiments have confirmed that $0.5 \% \mathrm{CMC}$ has no toxic side effects on experimental animals. ${ }^{20} \mathrm{HA}$ is a ubiquitous natural polysaccharide in the body with excellent physicochemical properties such as biodegradable, biocompatible, 
nontoxic, and nonimmunogenic characteristics. Accordingly, HA has been widely used for various medical applications such as arthritis treatment ${ }^{21}$, ophthalmic surgery ${ }^{22}$, drug delivery, ${ }^{23,24}$ and tissue engineering, ${ }^{25}$ So we did not set up a group for CIA treated with $0.5 \% \mathrm{CMC}$ or the blank hydrogel in the study. The total duration of drug administration was 21 days. Weight, ankle diameters, and AI scores of the experimental animals were measured every 3 days by two researchers.

\section{Histological Evaluation}

After drug intervention, the rats were euthanized by exsanguination under anesthesia and the right ankles were harvested for histological evaluation. The tissue was fixed, decalcified, and dehydrated before being embedded in paraffin. H\&E staining and saffron O-fixing green staining were performed to observe the histological changes. Hyperplasia of synovial tissue and blood vessels, infiltration of lymphocytes, and destruction of bone and cartilage were observed under a light microscope. The same sample was scored in terms of the Mankin's score (5 grades) based on observations of multiple visual fields, several times. ${ }^{26}$ The total semiquantitative score of the pathological injury of ankle joints was calculated. ${ }^{27}$

\section{Analysis Of The Expression Of Inflammatory Cytokines In Serum And Synovial Homogenate Supernatants By Enzyme-Linked Immunosorbent Assay (ELISA)}

Blood was taken from the canthus of the rats and centrifuged at $300 \times \mathrm{g}$ for $10 \mathrm{mins}$. Serum was collected and stored at $-80^{\circ} \mathrm{C}$. Synovial tissue was isolated and homogenized in PBS containing protease inhibitors (Applygen, China) and centrifuged, and the supernatant was collected. The levels of interleukin-1 beta (IL-1 $\beta$ ), interleukin-1 (IL-6), and TNF- $\alpha$ were determined using high-sensitivity ELISA kits according to the manufacturer's instructions (MultiScience, China).

\section{Statistical Analysis}

Statistical analysis was performed using SPSS V.22.0. Data were expressed as means \pm SD. Mann-Whitney U-test or ANOVA analyses were used to compare the differences between the groups. A value of $\mathrm{P}<0.05$ was considered to indicate a statistically significant difference.

\section{Results}

\section{Preparation And Characterization Of NanolGUR}

NanoIGUR with an IGUR : PVA weight ratio of 1:1 and a solvent : antisolvent ratio of 1:20 was prepared using the LAP technique. The TEM image revealed a spherical shape of NanoIGUR particles, ranging in size from 300 to $530 \mathrm{~nm}$ (Figure 3A). The average size of NanoIGUR, as determined using DLS, was $458 \mathrm{~nm}$ (Figure 3B), which was consistent with the TEM results. As shown in Figure $3 \mathrm{C}, E^{\prime}$ was consistently higher than $E^{\prime \prime}$ ( $E^{\prime}=$ storage modulus, $E^{\prime \prime}=$ loss modulus), and they exhibited a plateau in the entire frequency range of 0.01 to $10 \mathrm{~Hz}$ at $37^{\circ} \mathrm{C}$, suggesting that the hydrogels were robust and behaved as elastic solids.

\section{Vitro Drug Release}

The contents of IGUR in NanoIGUR and NanoIGURloaded hydrogel were measured using UV spectrophotometry. The drug-loading capacity of NanoIGUR was estimated to be $34.98 \%$. The loading efficiency further confirmed the combination of IGUR with the PVA excipient during the LAP process to form IGUR/PVA nanocomplexes. The comparison of the dissolution rates of raw IGUR and NanoIGUR prepared by the LAP method is shown in Figure 3D. Only $28.65 \%$ of raw IGUR was dissolved during the $72 \mathrm{hr}$ incubation period. In contrast, the LAP method effectively reduced the particle size of NanoIGUR, leading to a much higher dissolution rate of $80 \%$ and a very fast release equilibrium period of $24 \mathrm{hrs}$. The results proved that smaller particle size could improve the solubility owing to the increased surface area available for dissolution, as described by the Noyes-Whitney equation. ${ }^{28}$ Therefore, using the LAP technique is effective in improving the solubility and bioavailability of water-insoluble drugs.

NanoIGUR-loaded hydrogel was prepared by mixing the HA-AC solution containing NanoIGUR with the HSPEG-SH cross-linker solution, and this mixture was completely solidified after 5 mins, suggesting that the hydrogel formation time would be quick enough for in vivo applications. The drug dissolution profile of NanoIGUR-loaded hydrogel is shown in Figure 3D. IGUR was gradually released from the hydrogel matrix and the release could be sustained for $72 \mathrm{hrs}$, with a dissolution rate of $68 \%$. This suggests that the hydrogel composite can serve as a reservoir for sustained release of the encapsulated drug over a long term. 
A

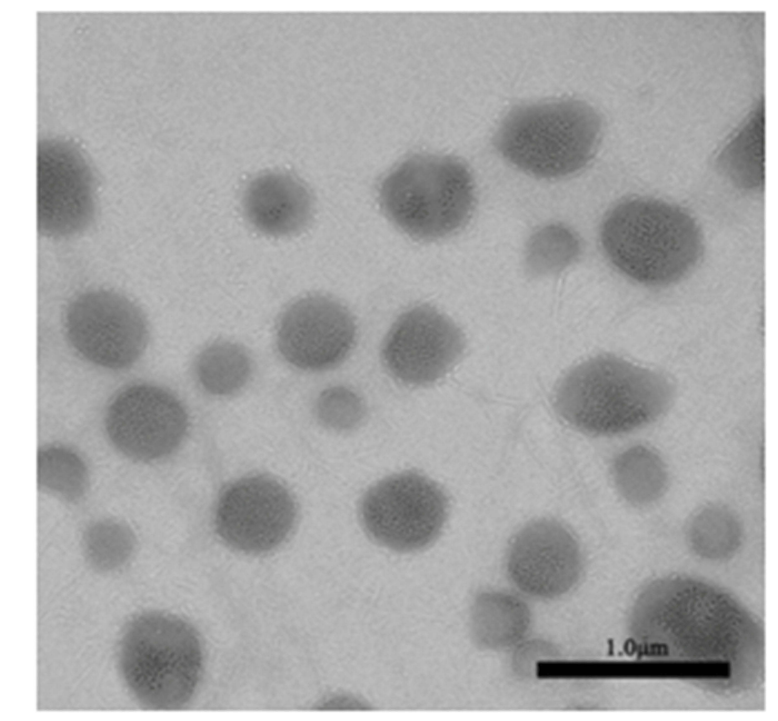

C

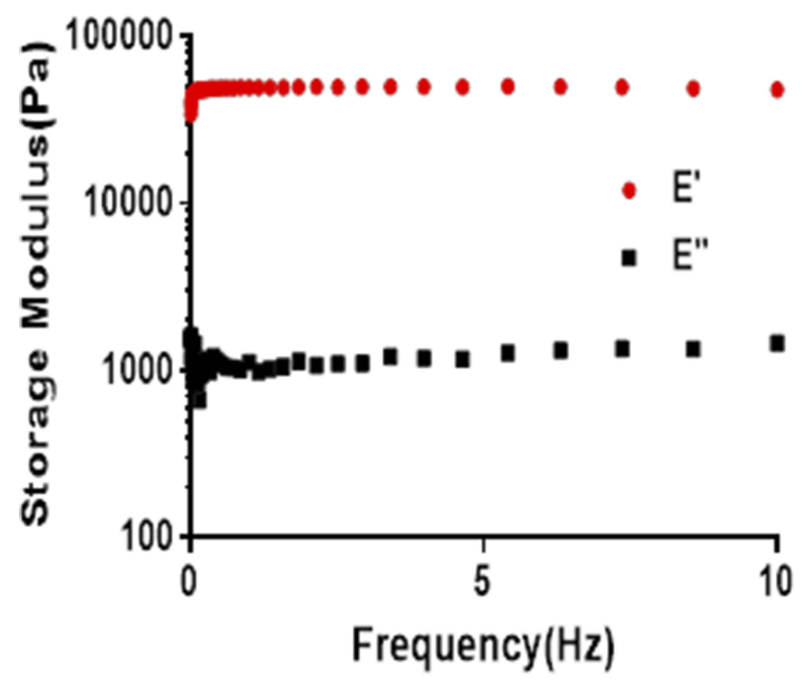

B

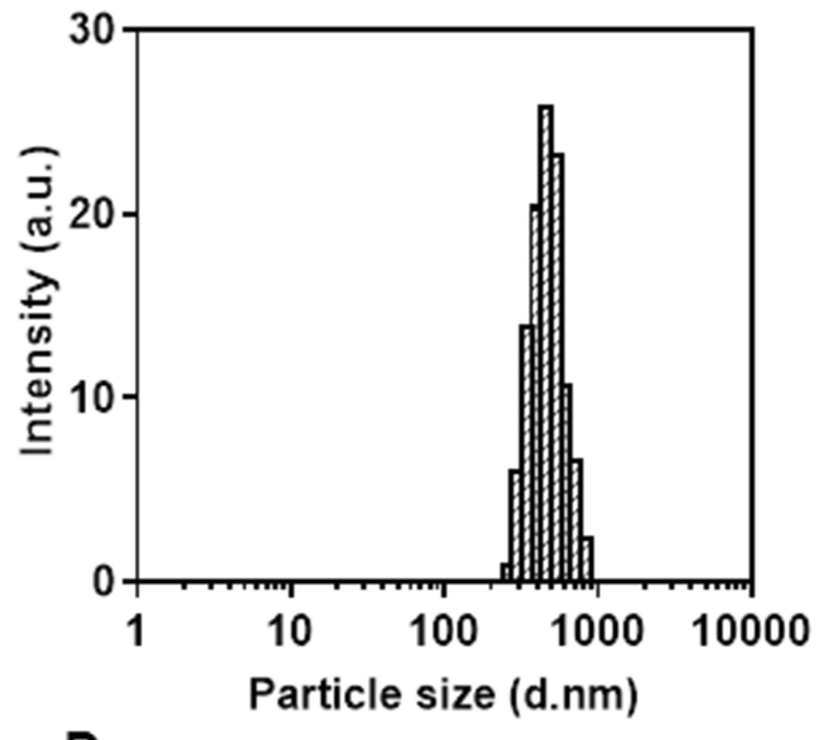

D

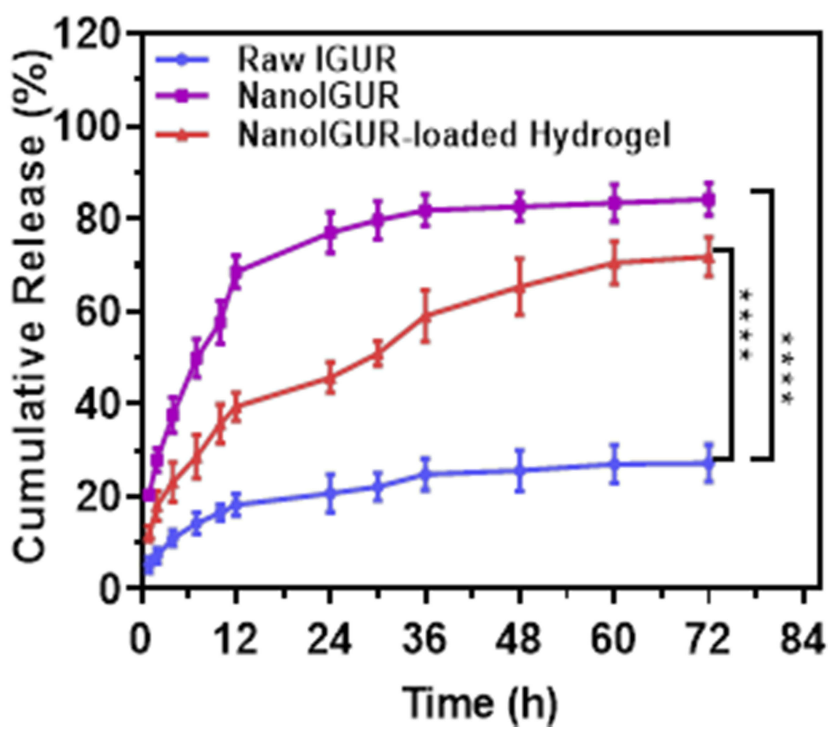

Figure 3 Characterization of NanolGUR and NanolGUR-loaded hydrogel. Transmission electron microscopy images of NanolGUR (A); Differential light scattering analyses of NanolGUR (B); DMTA of the hydrogel in the frequency range of $0.01 \mathrm{~Hz}$ to $10 \mathrm{~Hz}$ at $37^{\circ} \mathrm{C}(\mathbf{C})$; Dissolution profiles of raw IGUR, NanolGUR, and NanolGUR-loaded hydrogel (D). Data represent three independent experiments (mean $\pm S D$ ), ${ }^{* * * * p}<0.000$ I.

\section{Effects Of Different Dosage Forms Of IGUR On Proliferation, Migration, And Invasion Of RA-FLS}

In vitro drug release tests confirmed that the dissolution of the NanoIGUR-loaded hydrogel reached a plateau of release at 48 hrs. So, the in vitro cell proliferation, migration, and invasion experiments of RA-FLS were all conducted at $48 \mathrm{hrs}$. MTS assay has confirmed that NanoIGUR-loaded hydrogel could inhibit the proliferation of the RA-FLS in a dose-dependent manner. Furthermore, $160 \mu \mathrm{g} / \mathrm{mL}$ NanoIGUR-loaded hydrogel had better inhibitory effect than the same concentration of raw IGUR (relative cell proliferation ratio: $83.72 \pm 1.41 \%$ for raw IGUR; $74.25 \pm 1.17 \%$ for NanoIGUR-loaded hydrogel) and so did $320 \mu \mathrm{g} / \mathrm{mL}$ NanoIGUR-loaded hydrogel (relative cell proliferation ratio: $80.73 \pm 2.14 \%$ for raw IGUR; $65.25 \pm 2.31 \%$ for NanoIGUR-loaded hydrogel) (Figure 4A). Moreover, the different dosage forms of IGUR could inhibit migration and invasion of RA-FLS, with NanoIGUR-loaded hydrogel showing a better inhibitory effect than raw IGUR in the 160 and $320 \mu \mathrm{g} / \mathrm{mL}$ groups. For example, relative cell migration 
A

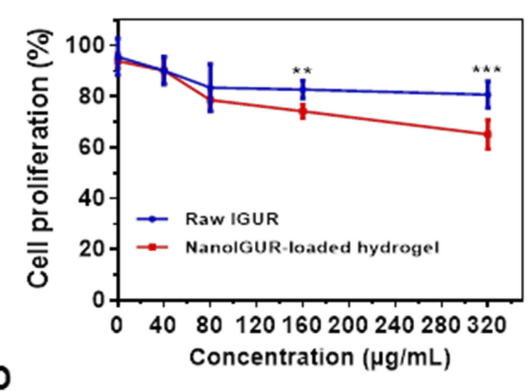

B

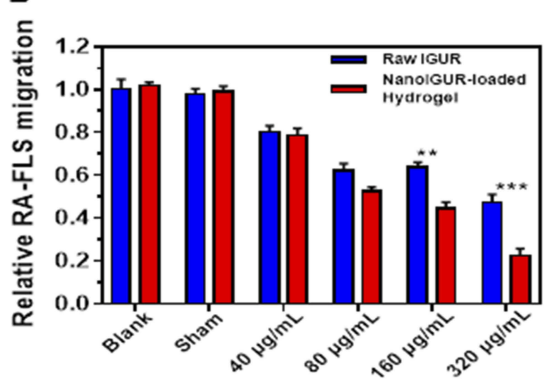

C

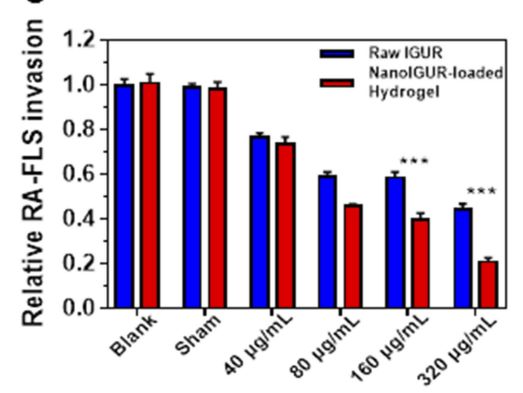

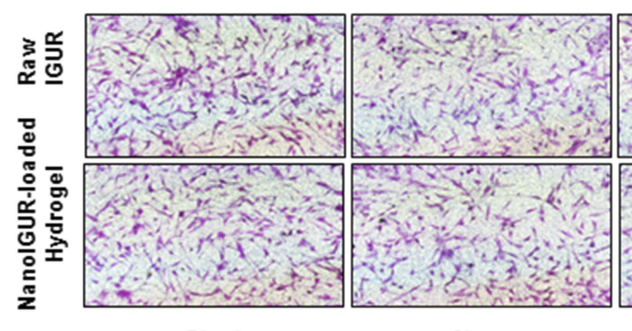
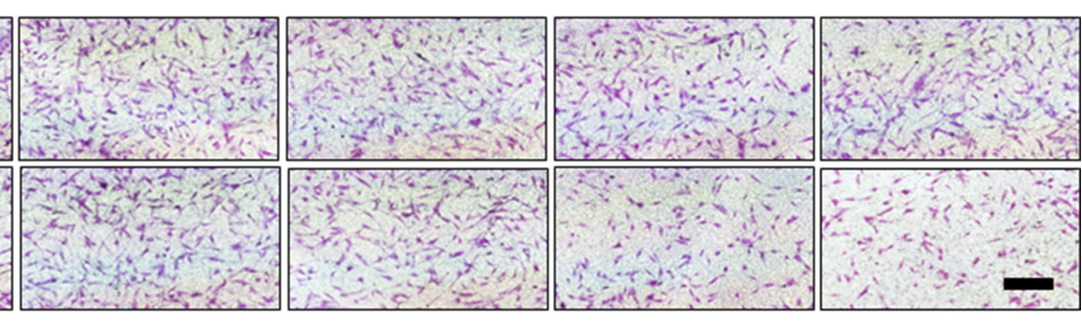

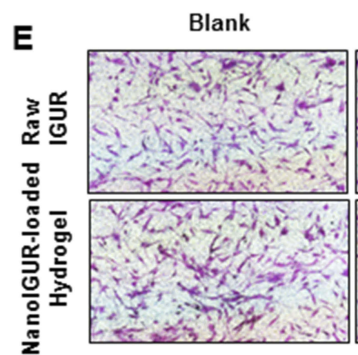

Blank

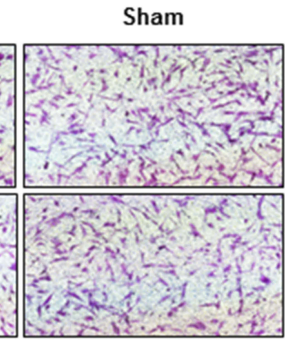

Sham
$40 \mu \mathrm{g} / \mathrm{mL}$

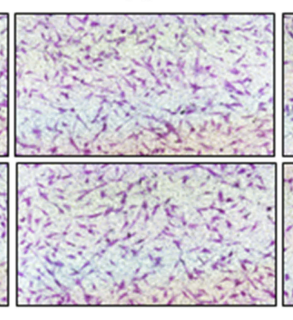

$40 \mu \mathrm{g} / \mathrm{mL}$

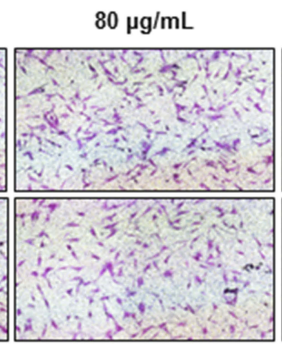

$80 \mu \mathrm{g} / \mathrm{mL}$
$160 \mu \mathrm{g} / \mathrm{mL}$

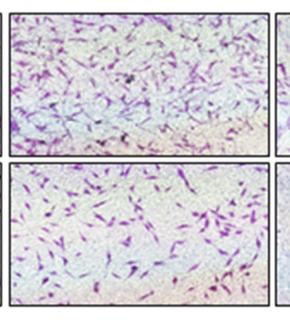

$160 \mu \mathrm{g} / \mathrm{mL}$
$320 \mu \mathrm{g} / \mathrm{mL}$

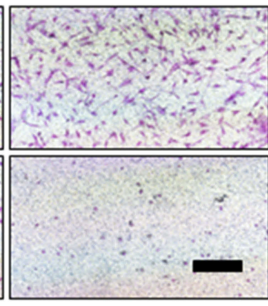

$320 \mu \mathrm{g} / \mathrm{mL}$

Figure 4 Effects of IGUR in different dosage forms on the proliferation, migration, and invasion of fibroblast-like synoviocytes (RA-FLS). Cell proliferation assay (A); cell migration assay (B); cell invasion assay (C); representative pictures for cell migration assay (D). Representative pictures for cell invasion assay (E). In the in vitro study, $10 \%$ FBS high glucose DMEM complete medium (complete medium) was used as the blank control, complete medium containing PBS was used as the sham control for the raw IGUR and the blank gel was used as the sham control for the NanolGUR-loaded hydrogel. Data represent three independent experiments. ${ }^{* *} p<0.0 \mathrm{I}, * * * p<0.00 \mathrm{I}, \mathrm{Scale}$ bar $=100 \mu \mathrm{m}$.

and invasion ratio for $320 \mu \mathrm{g} / \mathrm{mL}$ NanoIGUR-loaded hydrogel treated group were $22.42 \pm 1.96 \%$ and $20.74 \pm 1.16 \%$, respectively, while the relative cell migration and cell invasion ratio for raw IGUR group were $44.69 \pm 1.58 \%$ and $44.59 \pm 2.24 \%$, respectively. After excluding the effect of NanoIGUR-loaded hydrogel on cell proliferation, it still inhibit-ed the migration and invasion of RA-FLS (Figure 4B and C). Representative pictures are shown in Figure 4D and E.

\section{Plasma Concentration And Pharmacokinetic Parameters}

The plasma concentration-time curves for healthy rats after oral administration of raw IGUR and subcutaneous injection of NanoIGUR-loaded hydrogel $(10 \mathrm{mg} / \mathrm{kg})$ are shown in Figure 5A. The pharmacokinetic parameters were processed using the DAS 2.0 software. The $\mathrm{T}_{1 / 2}$ of NanoIGUR-loaded hydrogel was determined to be $13.7 \mathrm{hrs}$, which was more than the $\mathrm{T}_{1 / 2}$ of raw IGUR (3.7 hrs). The Cmax of NanoIGUR- loaded hydrogel was $5921.9 \mu \mathrm{g} / \mathrm{mL}$, which was lower than that of raw IGUR $(12,654.6 \mu \mathrm{g} / \mathrm{mL})$. The AUClast of NanoIGUR-loaded hydrogel and raw IGUR were 150,171.9 and $110,670 \mu \mathrm{g} / \mathrm{mL}$, respectively. The lower Cmax and delayed Tmax indicated the sustained-release characteristics of NanoIGUR-loaded hydrogel and the higher value of AUClast suggested better bioavailability. ${ }^{29-31}$

\section{Changes In Body Weight, Ankle Joint Diameter, And Al Scores After Drug Intervention In CIA Models}

According to the pharmacokinetic parameters, NanoIGURloaded hydrogel $(10 \mathrm{mg} / \mathrm{kg})$ was injected subcutaneously in rats every 3 days, and raw IGUR was given intragastrically daily for 21 days. The weight, ankle joint diameter, and AI scores were recorded every 3 days. As shown in Figure 5B, the AI scores in the NanoIGUR-loaded hydrogel group were significantly lower than in the CIA group on the 10th day of 

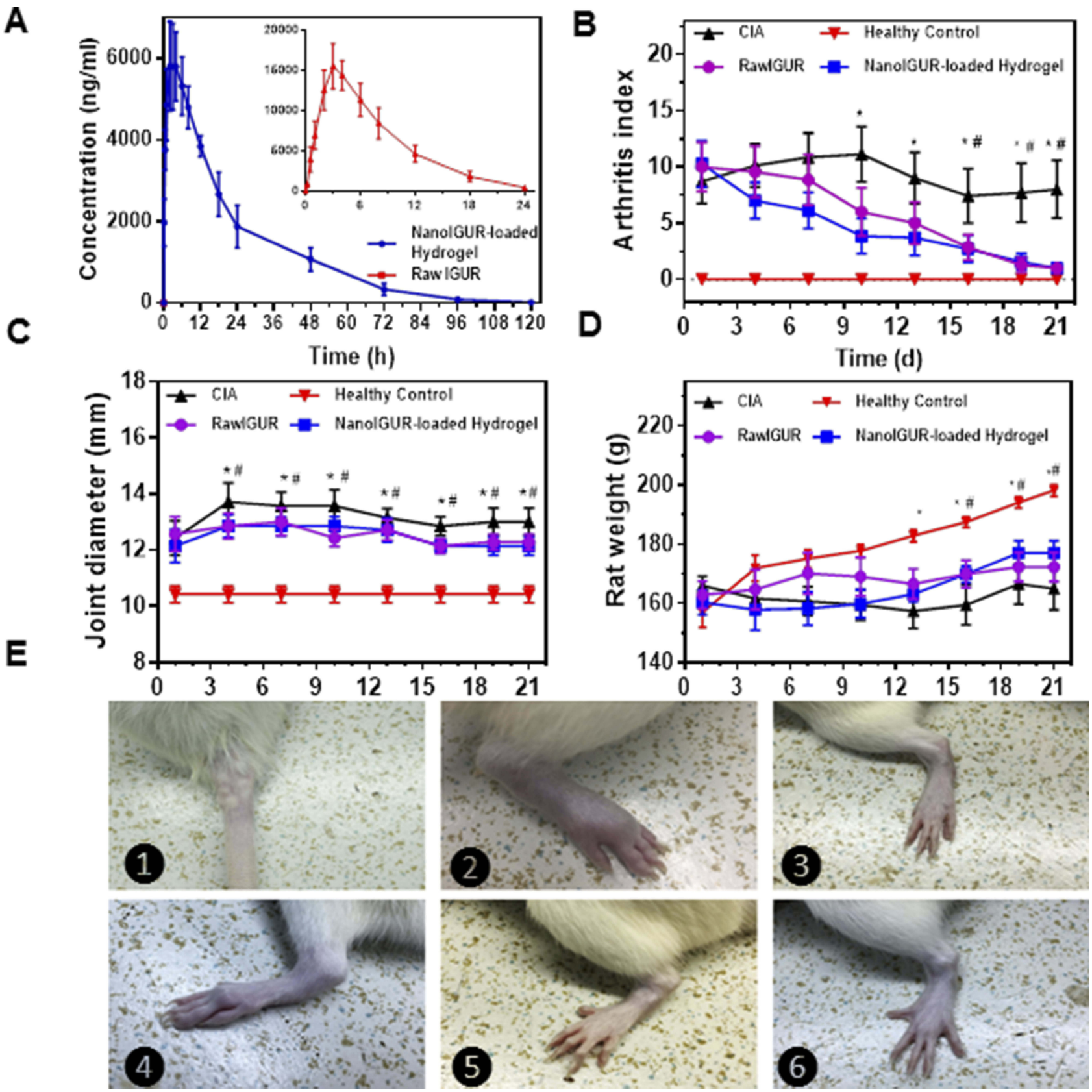

Figure 5 Changes in body weight, ankle joint diameter, and Al scores after drug intervention in $\mathrm{ClA}$ models. Comparision of drug concentration-time curve in healthy rats of raw IGUR and NanolGUR-loaded hydrogel (A); changes in the arthritis index of rats during drug intervention (B); changes in the joint diameter in rats during drug intervention (C); changes in the weight of rats during drug intervention (D); (E) $\mathbf{0}$ : Induction of collagen-induced arthritis (CIA) model by intradermal infection of bovine type II collagen emulsion; 2: Swollen joints of CIA model rats; (3: Ankle joints in healthy rats; 4: CIA model without any drug intervention showing obvious joint deformity 21 days after the onset; (5: CIA model rat treated with raw IGUR for 21 days; 6: CIA model rat treated with NanolGUR-loaded hydrogel for 21 days. Data represent three independent experiments (mean $\pm \mathrm{SD}$ ), $\mathrm{n}=7$ per group. ${ }^{*} p<0.05$, compared with NanolGUR-loaded hydrogel, \# $p<0.05$, compared with raw IGUR.

administration, and the difference was statistically significant. The AI scores in the raw IGUR group were significantly lower than in the CIA group on the 16th day of administration. From the 4 th day of administration, the mean diameters of the ankle joints in the NanoIGUR-loaded hydrogel and raw IGUR groups were significantly smaller than in the CIA group, and this was maintained until the end of the administration
(Figure 5C). On the 13th day of administration, the body weights in the NanoIGUR-loaded hydrogel and raw IGUR groups were significantly higher than in the CIA group, but were lower than in the healthy control group (Figure 5D). Representative pictures of rat ankle joints are shown in Figure 5E. These data indicate that NanoIGUR-loaded hydrogel and raw IGUR have similar therapeutic effects on CIA rats. 


\section{Histological Evaluation}

The H\&E-stained sections of ankle joints of rats were observed under a light microscope. In the control group, normal ankle joint structure, with smooth and intact articular cartilage surface and normal joint space, without synovial tissue proliferation or pannus formation was observed. In the CIA group, the ankle joint structure was severely destroyed, with hyperplastic synovial tissue, infiltrated inflammatory cells, pannus formation, and bone erosion were observed. The articular cartilage surface was damaged, the surface was stripped, and the joint space was narrowed. Compared with the CIA group, rat ankle joints from the raw IGUR and NanoIGUR-loaded hydrogel treated groups showed less infiltration of inflammatory cells in the synovial tissue and no obvious bone erosion (Figure 6A). The synovitis scores and Mankin's scores in the raw IGUR and NanoIGUR-loaded hydrogel groups were significantly lower $(\mathrm{P}<0.05)$ than in the CIA group, but there was no significant difference in the scores between the raw IGUR and NanoIGUR-loaded hydrogel groups (Figure $6 \mathrm{C}$ and D). The saffron O-fixing green staining showed that the basophilic cartilage stained red with saffron $\mathrm{O}$ and the acidophilic bone stained green or blue with the acid dye, thus distinguishing the cartilage tissue from the bone tissue. A complete cartilage band attached to the articular surface was observed in the healthy control group, whereas in the CIA group, the cartilage band on the articular surface almost disappeared, indicating that the entire cartilage layer was eroded. In the raw IGUR and NanoIGUR-loaded hydrogel groups, the cartilage on the articular surface was slightly thinner or wormlike, but the cartilage band remained intact or only a small amount of cartilage was eroded and lost, showing a slight discontinuity. Compared to the CIA group, the pathological changes in the ankle joint cartilage of rats in the raw IGUR and NanoIGUR-loaded hydrogel groups were significantly improved (Figure 6B). The percentage of cartilage area in each group was estimated using Image J software. Compared with the normal control group, the area of ankle cartilage in the CIA group was significantly
A

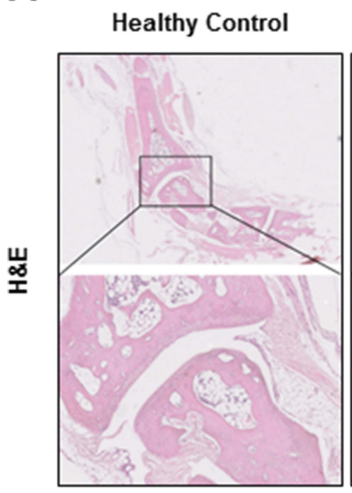

B

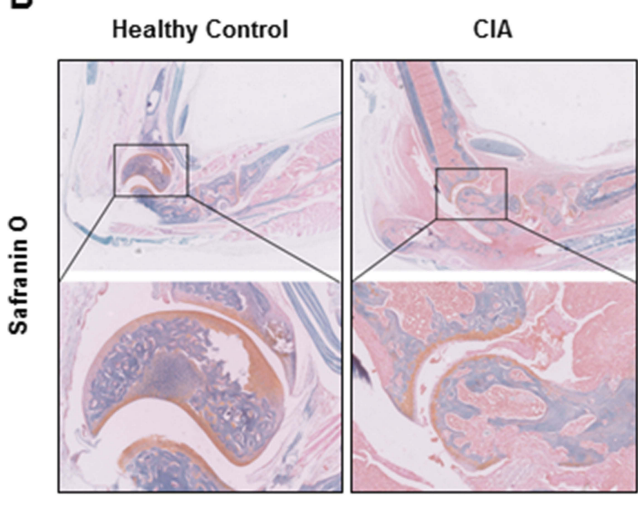

CIA

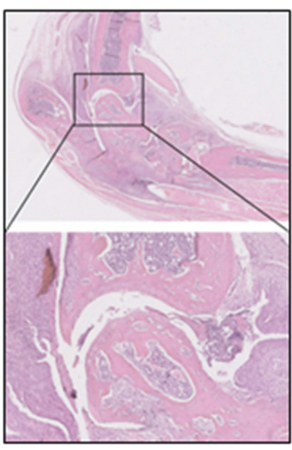

Raw IGUR

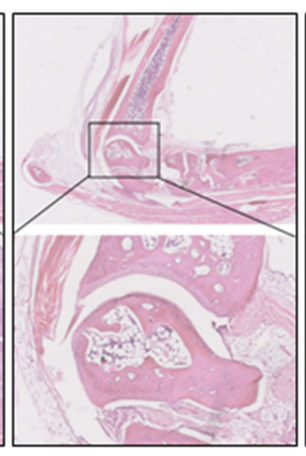

Raw IGUR

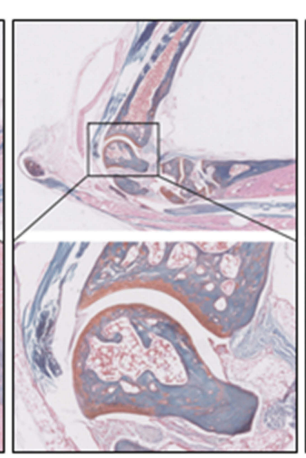

NanolGUR-loaded Hydrogel

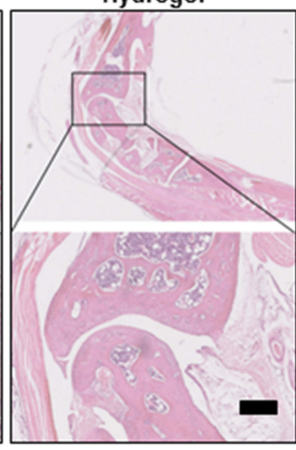

NanolGUR-loaded Hydrogel

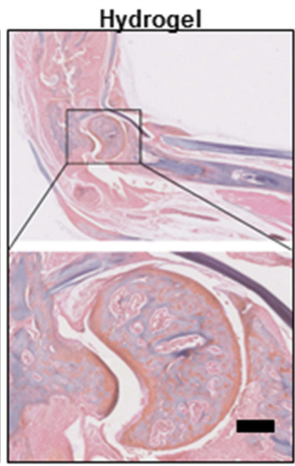

C
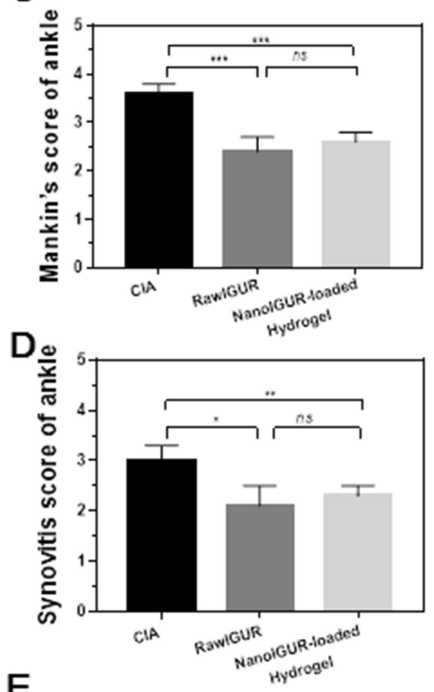

E

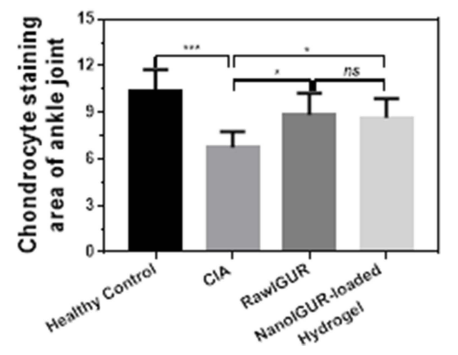

Figure 6 Results of histological evaluation. H\&E staining (A); saffron O-fixing green staining (B); Mankin's score of CIA rats (C); synovitis score of the CIA rats (D); percentage of cartilage area in each group $(\mathbf{E})$; healthy control $=$ healthy normal rats, $\mathrm{CIA}$ model $=\mathrm{CIA}$ rats with no intervention, raw IGUR= $\mathrm{CIA}$ rats treated with raw IGUR $10 \mathrm{mg} / \mathrm{kg}$ intragastrically, daily, NanolGUR-loaded hydrogel= CIA rats treated with NanolGUR-loaded hydrogel $10 \mathrm{mg} / \mathrm{kg}$ by subcutaneous injection, every 3 days. Data represent three independent experiments (mean $\pm \mathrm{SD}$ ), ${ }^{*} p<0.05, * * p<0.0 \mathrm{I}, * * *_{p}<0.00 \mathrm{I}$; ns, not significant; $\mathrm{n}=7$ per group, Scale bar $=200 \mu \mathrm{m}$. 
reduced $(\mathrm{P}<0.001)$. The area of ankle cartilage in the CIA rats increased significantly after drug intervention (raw IGUR and NanoIGUR-loaded hydrogel) $(\mathrm{P}<0.05)$, but there was no statistical difference between the raw IGUR and NanoIGUR-loaded hydrogel groups (Figure 6E).

\section{Expression Of Inflammatory Cytokines After Drug Intervention}

IL-1 $\beta$, IL- 6 , and TNF- $\alpha$ are crucial inflammatory cytokines in the process of inflammation in RA and are associated with the disease activity. ${ }^{32-34}$ Compared with the control group, IL-6, TNF- $\alpha$, and IL- $1 \beta$ levels in the serum of the CIA group were significantly upregulated. The serum levels of IL-6, TNF- $\alpha$, and IL- $1 \beta$ in the raw IGUR and NanoIGUR-loaded hydrogel groups were significantly lower than those in the CIA group, but there was no significant difference between the raw IGUR and NanoIGUR-loaded hydrogel groups (Figure 7A-C). Similar results were found in the synovial tissue homogenate supernatant (Figure 7D-F). Overall, these results demonstrate the efficacy of NanoIGUR-loaded hydrogel in the treatment of CIA rats. Subcutaneous injection of 10 $\mathrm{mg} / \mathrm{kg}$ NanoIGUR-loaded hydrogel every 3 days achieved the same therapeutic effect as the oral administration of 10 $\mathrm{mg} / \mathrm{kg}$ raw IGUR daily.

\section{Discussion}

Compared with the conventional administration, the sustained release of drugs has several advantages. It allows the maintenance of effective therapeutic concentration of the drug in body fluids for a longer time and reduces the times of administration, which is convenient for the patients. Moreover, it reduces the peak-valley of the drug concentration in the blood, reducing the side effects and improving the curative effects, and increases the stability of the drug. Sustained release of drugs also allows reduction in the dose and direct administration to specific target organs, which decreases the systemic side effects. Injectable hydrogels for in vivo localized drug delivery have achieved remarkable results in the development of new dosage forms that are not possible through traditional drug delivery and have shown huge prospects in the drug market, which has rapidly made them one of the most popular pharmaceutical dosage forms. ${ }^{35-37}$

Although the injectable hydrogel in vivo localization dosage form has many advantages, there are presently some limitations in its use. ${ }^{38-40}$ The even distribution
A

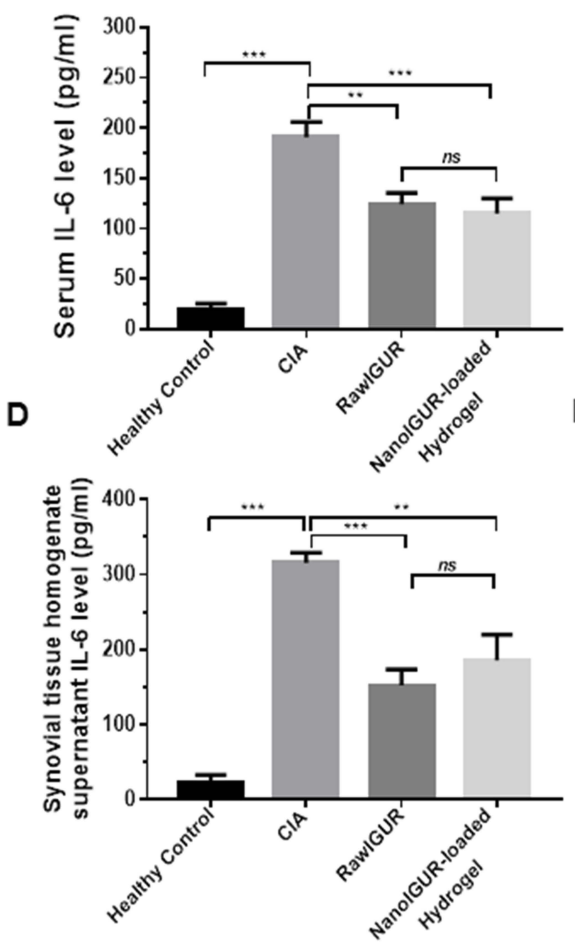

B

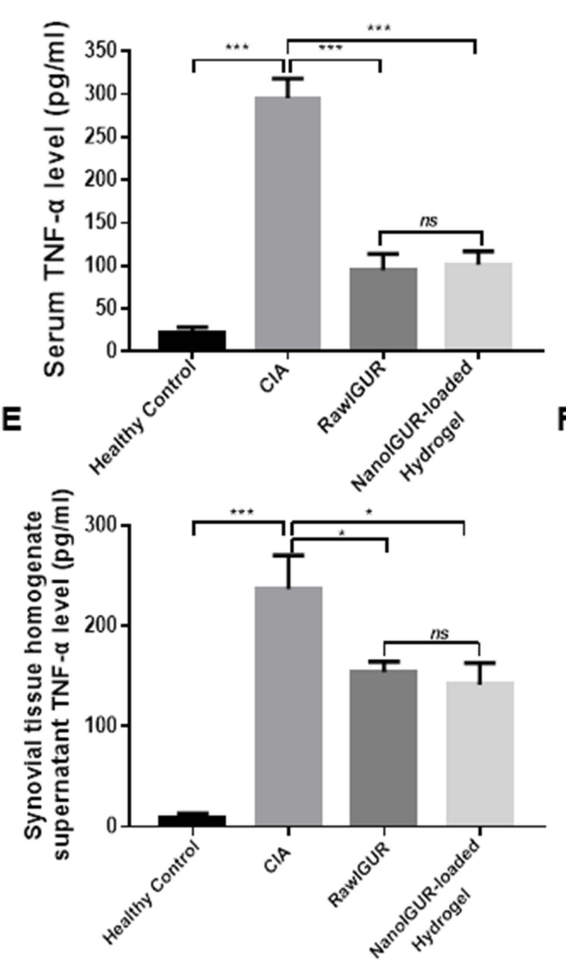

C

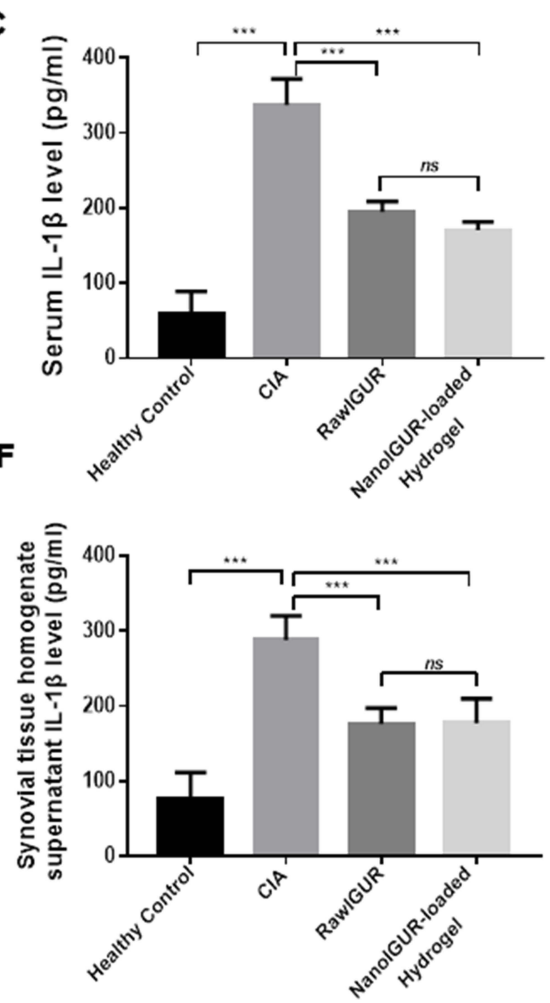

Figure 7 Analysis of the expression of inflammatory cytokines in serum and synovial homogenate supernatants by enzyme-linked immunosorbent assay (ELISA). Serum levels of IL-6 (A), TNF- $\boldsymbol{\alpha}(\mathbf{B})$, and IL-I $\boldsymbol{\beta}(\mathbf{C})$. Levels of IL-6 (D), TNF- $\boldsymbol{\alpha}(\mathbf{E})$, and IL-I $\boldsymbol{\beta}(\mathbf{F})$ in the synovial tissue homogenate supernatant. Data represent three independent experiments (mean $\pm \mathrm{SD}$ ), ${ }^{*} p<0.05, *_{p}<0.0 \mathrm{I}$, ${ }^{* * *} p<0.00 \mathrm{I}$, ns, not significant. $\mathrm{n}=7$ per group. 
and stabilization of water-insoluble drugs in the hydrogel matrix is a key issue that needs to be resolved for the construction of hydrogel dosage forms. Therapeutic drugs are mostly hydrophobic molecules, ${ }^{41-44}$ and the hydrogel has a certain network pore size. Therefore, only when a hydrophobic drug can easily be dispersed into particles, having a size larger than that of the hydrogel pore, can it be uniformly and stably restricted. In the hydrogel matrix, it is further released from the hydrogel uniformly and constantly by passive diffusion of molecules and by the degradation of the hydrogel host material. The liquid antisolvent technology is a new method for preparing nanomicron powders, which has been proposed in recent years, especially for the preparation of nanomicron drugs, and has shown prospects for broad application. ${ }^{45}$ Based on the molecular mixing theory, the fast molecular mixing characteristics in the liquid environment can effectively and accurately control the synthesis of drug particles. Thus, drug nanoparticles with high nanometer size and uniform particle size can be synthesized, and nanomedicines with excellent dispersion properties, high dissolution, and high bioavailability in the aqueous phase can be prepared by controlling the particle size and using the dispersant.

IGUR is used in the treatment of RA, but gastrointestinal side effect caused by conventional oral dosage forms limits its application. For the first time, we tried to develop a new dosage form of IGUR to alleviate its side effects and to satisfy the needs of clinical therapy. As shown in Figure 2, first, NanoIGUR with high aqueous phase dispersibility was synthesized using the LAP method. Subsequently, we prepared HA-ADH, with amino group from $\mathrm{HA}$ and $\mathrm{ADH}$, by condensation reaction, and then reacted it with $\mathrm{N}$-acryloyloxysuccinimide to obtain a double bond on the segment of HA-AC. Furthermore, HA-AC was completely dissolved in PBS buffer and sequentially mixed with NanoIGUR and the crosslinking agent PEG (Thiol) $)_{2}$ (HS-PEG-SH) to prepare a hydrogel precursor solution. After subcutaneous injection, hydrogels encapsulating NanoIGUR were formed in situ by Michael addition reaction. ${ }^{46-48}$

After the preparation of the new dosage form of NanoIGUR-loaded hydrogel, we first determined its effects on the proliferation, migration, and invasion of RA-FLS. The NanoIGUR-loaded hydrogel showed better inhibitory effect than raw IGUR generally in the 160 and $320 \mu \mathrm{g} / \mathrm{mL}$ groups, which indicated that the NanoIGUR-loaded hydrogel improved the solubility of the water-insoluble IGUR and might act as a new drug-delivery system for future clinical applications. Thereafter, the plasma concentration and pharmacokinetic parameters of raw IGUR and NanoIGUR-loaded hydrogel were tested in healthy rats. The pharmacokinetics of the NanoIGUR-loaded hydrogel indicated that intermittent subcutaneous administration is feasible, which might improve the compliance of patients by reducing the number of administrations and the risk of gastrointestinal side effects. According to the pharmacokinetic parameters, NanoIGUR-loaded hydrogel $(10 \mathrm{mg} / \mathrm{kg})$ was injected subcutaneously in CIA models of rats every 3 days, and raw IGUR was given intragastrically, daily. Changes in body weight, ankle joint diameter, and AI score after 21 days of drug intervention indicated that NanoIGUR-loaded hydrogel and raw IGUR had similar therapeutic effects on the CIA rat models. By changing the route of administration, the same effect and longer interval of administration could be achieved, which shows that the new drug-delivery system - the NanoIGUR-loaded hydrogel, is suitable for animal experiments and may have good prospects for clinical applications in the future. Histological evaluation has further confirmed that both raw IGUR and NanoIGUR-loaded hydrogel could effectively reduce the destruction of cartilage and bone in the ankle joints of CIA rats and could reduce the proliferation of synovium and infiltration of inflammatory cells in the synovial tissue.

IL-1 $\beta$, IL-6, and TNF- $\alpha$ are crucial inflammatory cytokines in the process of inflammation in RA and are associated with the disease activity. The expression levels of inflammatory cytokines (IL-1 $\beta$, IL- 6 , and TNF- $\alpha$ ) in the serum and supernatant of the synovial tissue homogenate demonstrate the efficacy of NanoIGUR-loaded hydrogel in the treatment of CIA rats. Subcutaneous injection of 10 $\mathrm{mg} / \mathrm{kg}$ NanoIGUR-loaded hydrogel every 3 days achieved the same therapeutic effect as the oral administration of 10 $\mathrm{mg} / \mathrm{kg}$ raw IGUR daily. By changing the dosage form of IGUR into NanoIGUR-loaded hydrogel, we reduced the dosage of raw drugs, achieved long-term and sustained release, and acquired the same therapeutic effect. Although it is not ideal to apply the three-day administration of NanoIGUR-loaded hydrogel in clinical application, our research provides a kind of new thinking and method. Further improvements in the composition of hydrogels may extend the interval of administration.

\section{Conclusion}

RA is a common systemic autoimmune disease characterized by erosive synovitis, which leads to the damage of the 
joint architecture and consequent disability. Early treatment with DMARDs is the key to prevent joint destruction and improve prognosis. As a novel type of DMARD, IGUR is effective in treating RA by inhibiting the secretion of polyclonal immunoglobulin and production of cytokines. $^{49}$ At present, only oral-administered IGUR tablets are available because of its hydrophobic properties. The prevalence of gastrointestinal side effects limits the use of IGUR in clinical practice. The designing of a sustained and localized drug-delivery system for subcutaneous or intra-articular administration may increase its use by improving the bioavailability and by preventing gastrointestinal irritation. To achieve this goal, a new drugdelivery system, the nanoloaded hydrogel, was developed, and in vitro and in vivo experiments confirmed it to be an effective drug-delivery system. NanoIGUR-loaded hydrogel released IGUR in a sustained manner with long-acting time, and its subcutaneous injection achieved similar efficacy in treating CIA rats as did the oral administration of raw IGUR. However, the biocompatibility, biodegradability, and clinical safety of the new hydrogel carrier need to be further studied in the future. Overall, the NanoIGURloaded hydrogel may serve as a new drug-delivery system for specific DMARDs with broad prospects for clinical application.

\section{Acknowledgments}

We gratefully acknowledge the helpful discussions with Professor Xueqing Wang on the animal pharmacokinetic experiments. This work was financially supported by the National Natural Science Foundation of China (Grant 21476018, Grant 21622601, Grant 51972339, and Grant 81571573), the National Key R\&D Program of China (Grant 2015CB932101), and the Open Project Program of the State Key Laboratory of Organic-Inorganic Composites, Beijing University of Chemical Technology (Grant oic201901010).

\section{Disclosure}

The authors report no conflicts of interest in this work.

\section{References}

1. Mahajan TD, Mikuls TR. Recent advances in the treatment of rheumatoid arthritis. Curr Opin Rheumatol. 2018;30(3):231-237. doi:10.1097/BOR.0000000000000496

2. Komatsu N, Takayanagi H. [Bone and cartilage destruction in rheumatoid arthritis]. Clin Calcium. 2012;22(2):179-185. doi:CliCa1202179185
3. Corbacho MI, Dapueto JJ. Assessing the functional status and quality of life of patients with rheumatoid arthritis. Rev Bras Reumatol. 2010;50(1):31-43. doi:10.1590/S0482-50042010000100004

4. Goekoop-Ruiterman YP, Huizinga TW. Rheumatoid arthritis: can we achieve true drug-free remission in patients with RA? Nat Rev Rheumatol. 2010;6(2):68-70. doi:10.1038/nrrheum.2009.253

5. Osiri M, Deesomchok U, Tugwell P. Disease activity and functional changes of RA patients receiving different DMARDs in clinical practice. Clin Rheumatol. 2006;25(5):721-727. doi:10.1007/s10067-005-0155-4

6. Rubbert-Roth A, Finckh A. Treatment options in patients with rheumatoid arthritis failing initial TNF inhibitor therapy: a critical review. Arthritis Res Ther. 2009;11 Suppl 1: S1.doi:10.1186/ar2666

7. Day R. Adverse reactions to TNF-alpha inhibitors in rheumatoid arthritis. Lancet. 2002;359(9306):540-541. doi:10.1016/S0140-6736(02)07718-8

8. Chou CT. The high cost of anti-TNFalpha drugs for rheumatoid arthritis: can a low-price product be developed in the future? $J$ Chin Med Assoc. 2012;75(2):51-53. doi:10.1016/j.jcma.2011.12.012

9. Mello SB, Tavares ER, Bulgarelli A, Bonfa E, Maranhao RC. Intraarticular methotrexate associated to lipid nanoemulsions: anti-inflammatory effect upon antigen-induced arthritis. Int $J$ Nanomedicine. 2013;8:443-449. doi:10.2147/IJN.S29392

10. Prabhu P, Shetty R, Koland M, et al. Investigation of nano lipid vesicles of methotrexate for anti-rheumatoid activity. Int $J$ Nanomedicine. 2012;7:177-186. doi:10.2147/IJN.S25310

11. Mucke HA. Iguratimod: a new disease-modifying antirheumatic drug. Drugs Today (Barc). 2012;48(9):577-586. doi:10.1358/dot.2012.48. 9.1855758

12. Li J, Mao H, Liang Y, et al. Efficacy and safety of iguratimod for the treatment of rheumatoid arthritis. Clin Dev Immunol. 2013;2013:310628. doi: $10.1155 / 2013 / 310628$

13. Jinno J, Kamada N, Miyake M, et al. Effect of particle size reduction on dissolution and oral absorption of a poorly water-soluble drug, cilostazol, in beagle dogs. J Control Release. 2006; 111 (1-2): 56-64. doi:10.1016/j.jconrel.2005.11.013

14. Tokatlian T, Cam C, Siegman SN, Lei Y, Segura T. Design and characterization of microporous hyaluronic acid hydrogels for in vitro gene transfer to mMSCs. Acta Biomater. 2012;8(11):39213931. doi:10.1016/j.actbio.2012.07.014

15. Kay J, Upchurch KS. ACR/EULAR 2010 rheumatoid arthritis classification criteria. Rheumatology (Oxford). 2012;51 Suppl 6: vi5-vi9. doi:10.1093/rheumatology/kes279

16. Zimmermann T, Kunisch E, Pfeiffer R, et al. Isolation and characterization of rheumatoid arthritis synovial fibroblasts from primary culture-primary culture cells markedly differ from fourth-passage cells. Arthritis Res. 2001;3(1):72-76. doi:10.1186/ar142

17. Zhou T, Ding L, Li X, et al. Determination of iguratimod in rat plasma by high performance liquid chromatography: method and application. Biomed Chromatogr. 2008;22(3):260-264. doi:10.1002/bmc.921

18. Hirose J, Tanaka S. [Animal models for bone and joint disease. CIA, CAIA model]. Clin Calcium. 2011;21(2):253-259. CliCa1102253259

19. Courtenay JS, Dallman MJ, Dayan AD, Martin A, Mosedale B. Immunisation against heterologous type II collagen induces arthritis in mice. Nature. 1980;283(5748):666-668. doi:10.1038/283666a0

20. Iwanaga Y, Kihara Y, Takenaka H, Kita T. Down-regulation of cardiac apelin system in hypertrophied and failing hearts: possible role of angiotensin II-angiotensin type 1 receptor system. J Mol Cell Cardiol. 2006;41(5):798-806. doi:10.1016/j.yjmcc.2006.07.004

21. Balazs EA, Denlinger JL. Viscosupplementation: a new concept in the treatment of osteoarthritis. J Rheumatol Suppl. 1993;39:3-9.

22. Balazs EA. Viscosupplementation for treatment of osteoarthritis: from initial discovery to current status and results. Surg Technol Int. 2004;12:278-289.

23. Hahn SK, Kim SJ, Kim MJ, Kim DH. Characterization and in vivo study of sustained-release formulation of human growth hormone using sodium hyaluronate. Pharm Res. 2004;21(8):1374-1381. doi:10.1023/b:pham.0000036910.41224.de 
24. Kim SJ, Hahn SK, Kim MJ, Kim DH, Lee YP. Development of a novel sustained release formulation of recombinant human growth hormone using sodium hyaluronate microparticles. J Control Release. 2005;104(2):323-335. doi:10.1016/j.jconrel.2005.02.012

25. Ohri R, Hahn SK, Hoffman AS, Stayton PS, Giachelli CM. Hyaluronic acid grafting mitigates calcification of glutaraldehydefixed bovine pericardium. J Biomed Mater Res A. 2004;70(2):328334. doi:10.1002/jbm.a.30088

26. Kuroki H, Nakagawa Y, Mori K, et al. Acoustic stiffness and change in plug cartilage over time after autologous osteochondral grafting: correlation between ultrasound signal intensity and histological score in a rabbit model. Arthritis Res Ther. 2004;6(6):R492-504. doi:10. $1186 /$ ar1219

27. Krenn V, Morawietz L, Burmester GR, et al. Synovitis score: discrimination between chronic low-grade and high-grade synovitis. Histopathology. 2006;49(4):358-364. doi:10.1111/j.1365-2559.2006.02508.x

28. Horn D, Rieger J. Organic nanoparticles in the aqueous phase-theory, experiment, and use. Angew Chem Int Ed Engl. 2001;40(23):4330-4361. doi:10.1002/1521-3773(20011203)40:23<4330::AID-ANIE4330>3.0. $\mathrm{CO} ; 2-\mathrm{W}$

29. Silber BM, Rao S, Fife KL, et al. Pharmacokinetics and metabolism of 2-aminothiazoles with antiprion activity in mice. Pharm Res. 2013;30(4):932-950. doi:10.1007/s11095-012-0912-4

30. Rausch-Derra LC, Rhodes L, Freshwater L, Hawks R. Pharmacokinetic comparison of oral tablet and suspension formulations of grapiprant, a novel therapeutic for the pain and inflammation of osteoarthritis in dogs. $J$ Vet Pharmacol Ther. 2016;39(6):566-571. doi:10.1111/jvp. 12306

31. Portoles A, Almeida S, Terleira A, et al. Truncated AUC in the evaluation of fluconazole bioequivalence. A cross-over, randomised, open-label study in healthy volunteers. Arzneimittelforschung. 2004;54(11):752-756. doi:10.1055/s-0031-1297032

32. Zhao PW, Jiang WG, Wang L, Jiang ZY, Shan YX, Jiang YF. Plasma levels of IL-37 and correlation with TNF-alpha, IL-17A, and disease activity during DMARD treatment of rheumatoid arthritis. PLoS One. 2014;9(5):e95346. doi:10.1371/journal.pone.0095346

33. Houssiau FA, Devogelaer JP, Van Damme J, de Deuxchaisnes CN, Van Snick J. Interleukin-6 in synovial fluid and serum of patients with rheumatoid arthritis and other inflammatory arthritides. Arthritis Rheum. 1988;31(6):784-788. doi:10.1002/art.1780310614

34. Lettesjo H, Nordstrom E, Strom H, et al. Synovial fluid cytokines in patients with rheumatoid arthritis or other arthritic lesions. Scand J Immunol. 1998;48(3):286-292. doi:10.1046/j.1365-3083. 1998.00399.x

35. Lee F, Chung JE, Kurisawa M. An injectable hyaluronic acid-tyramine hydrogel system for protein delivery. $J$ Control Release. 2009;134(3):186-193. doi:10.1016/j.jconrel.2008.11.028

36. Nakaji-Hirabayashi T, Kato K, Iwata H. Hyaluronic acid hydrogel loaded with genetically-engineered brain-derived neurotrophic factor as a neural cell carrier. Biomaterials. 2009;30(27):4581-4589. doi:10.1016/j.biomaterials.2009.05.009
37. Lei Y, Huang S, Sharif-Kashani P, Chen Y, Kavehpour P, Segura T. Incorporation of active DNA/cationic polymer polyplexes into hydrogel scaffolds. Biomaterials. 2010;31(34):9106-9116. doi:10.1016/j. biomaterials.2010.08.016

38. Liu J, Qi C, Tao K, et al. Sericin/dextran injectable hydrogel as an optically trackable drug delivery system for malignant melanoma treatment. ACS Appl Mater Interfaces. 2016;8(10):6411-6422. doi:10.1021/ acsami.6b00959

39. Liu M, Song X, Wen Y, Zhu JL, Li J. Injectable thermoresponsive hydrogel formed by alginate-g-poly( $\mathrm{N}$-isopropylacrylamide) that releases doxorubicin-encapsulated micelles as a smart drug delivery system. ACS Appl Mater Interfaces. 2017;9(41):35673-35682. doi:10. 1021/acsami.7b12849

40. Thambi T, Li Y, Lee DS. Injectable hydrogels for sustained release of therapeutic agents. J Control Release. 2017;267:57-66. doi:10.1016/ j.jconrel.2017.08.006

41. Kang L, Gao Z, Huang W, Jin M, Wang Q. Nanocarrier-mediated codelivery of chemotherapeutic drugs and gene agents for cancer treatment. Acta Pharm Sin B. 2015;5(3):169-175. doi:10.1016/j. apsb.2015.03.001

42. Rabinow BE. Nanosuspensions in drug delivery. Nat Rev Drug Discov. 2004;3(9):785-796. doi:10.1038/nrd1494

43. Muller RH, Jacobs C, Kayser O. Nanosuspensions as particulate drug formulations in therapy. Rationale for development and what we can expect for the future. Adv Drug Deliv Rev. 2001;47(1):3-19. doi:10.1016/S0169-409X(00)00118-6

44. Hu J, Johnston KP, Williams RO, 3rd. Nanoparticle engineering processes for enhancing the dissolution rates of poorly water soluble drugs. Drug Dev Ind Pharm. 2004;30(3):233-245. doi:10.1081/DDC120030422

45. Cushing BL, Kolesnichenko VL, O'Connor CJ. Recent advances in the liquid-phase syntheses of inorganic nanoparticles. Chem Rev. 2004;104(9):3893-3946. doi:10.1021/cr030027b

46. Elbert DL, Pratt AB, Lutolf MP, Halstenberg S, Hubbell JA. Protein delivery from materials formed by self-selective conjugate addition reactions. J Control Release. 2001; 76 (1-2): 11-25. doi:10.1016/ S0168-3659(01)00398-4

47. Shu XZ, Ahmad S, Liu Y, Prestwich GD. Synthesis and evaluation of injectable, in situ crosslinkable synthetic extracellular matrices for tissue engineering. J Biomed Mater Res A. 2006;79(4):902-912. doi:10.1002/jbm.a.30831

48. Lei Y, Gojgini S, Lam J, Segura T. The spreading, migration and proliferation of mouse mesenchymal stem cells cultured inside hyaluronic acid hydrogels. Biomaterials. 2011;32(1):39-47. doi:10.1016/ j.biomaterials.2010.08.103

49. Tanaka K, Yamamoto T, Aikawa Y, et al. Inhibitory effects of an antirheumatic agent T-614 on immunoglobulin production by cultured B cells and rheumatoid synovial tissues engrafted into SCID mice. Rheumatology (Oxford). 2003;42(11):1365-1371. doi:10.1093/rheumatology/keg381
International Journal of Nanomedicine

\section{Publish your work in this journal}

The International Journal of Nanomedicine is an international, peerreviewed journal focusing on the application of nanotechnology in diagnostics, therapeutics, and drug delivery systems throughout the biomedical field. This journal is indexed on PubMed Central, MedLine, CAS, SciSearch ${ }^{\mathbb{R}}$, Current Contents ${ }^{\mathbb{R}} /$ Clinical Medicine, $^{2}$
Journal Citation Reports/Science Edition, EMBase, Scopus and the Elsevier Bibliographic databases. The manuscript management system is completely online and includes a very quick and fair peer-review system, which is all easy to use. Visit http://www.dovepress.com/ testimonials.php to read real quotes from published authors. 